\title{
Revisiting Technology and Flow: A Call for an Alternative Perspective and Directions for the Future
}

\author{
Christopher B. Califf \\ Western Washington University \\ Christopher.Califf@wwu.edu
}

\author{
T.S. Stumpf \\ Humboldt State University \\ Tyler.Stumpf@humboldt.edu
}

\author{
Joshua J. Frye \\ Humboldt State University \\ Joshua.Frye@ humboldt.edu
}

\begin{abstract}
Employee engagement is critical to individual wellbeing and organizational performance. The concept of flow has been explored as a marker for such engagement. Yet, an understanding of the role technology plays in employees experiencing flow is not well understood. In this paper, we theorize an alternative viewpoint of flow and technology, which we coin "technoflow." We do so by critically examining the assumptions within existing IS/flow literature, and propose a research agenda that adopts a relational ontology so that IS researchers can identify several sociomaterial conditions and practices related to how employees experience flow. We explain how researchers can draw on technoflow through four central themes: (1) control; (2) attention; (3) curiosity; and (4) intrinsic interest. We provide guidance about how to incorporate technoflow into two contemporary IS theories: media synchronicity theory and technostress. This intervention offers promising theoretical development and knowledge applications for IS researchers and practitioners alike.
\end{abstract}

\section{Introduction}

Facilitating employee engagement should be a priority for leaders and managers. Unfortunately, employee engagement levels remain low. For example, a 2017 Gallup poll found only $34 \%$ of U.S. employees were engaged in their work, with the remaining 66\% self-reporting as either "not engaged" or "actively disengaged" [12]. These findings on lack of engagement suggest that most employees are functioning at a fraction of their true potential. In most organizations today, information technology (IT) plays a major role in facilitating how employees accomplish a variety of tasks. It follows that technology in organizations therefore needs to be designed and used so that employees can become effectively engaged in their work. One concept that has played a central role in identifying and understanding the role technology plays in why and how individuals become engaged in work is the concept of flow $[1,35]$.

Developed by Csikszentmihalyi [6], flow encompasses "the state in which people are so involved in an activity that nothing else seems to matter" [7]. Flow has been acknowledged as a valuable concept in the Information Systems (IS) discipline over the last few decades [11, 26]. For example, researchers have identified the importance of flow in web design, intention to use technology, elearning, and usability, among others [1]. However, a line of recent scholarship has argued that the current state of flow research in the IS discipline is problematic [5, 26]. For example, in a discussion on the measurement of flow in IS research, Choi et al. [5] assert that flow is "too broad and ill-defined due to the numerous ways it has been operationalized, tested, and applied." Moreover, Novak et al. [29] mention that there are 13 flow constructs used across IS research, several of which do not seem to achieve a conceptual match with the concept of flow.

To address this "broad and ill-defined" view of flow, IS researchers have recently revisited and reviewed the literature on flow in relation to technology. For example, Rissler et al. [39] articulate four streams of flow research in IS, which are used to provide an integrative theoretical framework of flow. Likewise, Mahnke et al. [25] attempt to instantiate several empirically measurable concepts associated with flow in IS (i.e., absorption, fluency, and enjoyment), and then test these constructs as influencing one's continuance intention. In this sense, there seems to be rejuvenation and renewed interest in understanding the interplay of flow and technology in the IS domain.

While these literature reviews have contributed to a renewed interest in technology and flow, and provide valuable directions for the future, the frameworks used are primarily based on ontological and 
epistemological assumptions embedded in perspectives of technology that are often classified as "immaterial" or as an "exogenous force" [30, 32, 40]. In this sense, the calls for further research on flow, and the research designs embedded in them, are primarily based on variance logic that rely on statistical methods to test causal relationships among variables. Moreover, the calls do not articulate how researchers and managers can identify patterns associated with employees using technology and their experiences (or lack thereof) with flow.

The goal of this paper is to take a first step to answer a call by Csikszentmihalyi [8], who underscored that in the context of organizations, managers should strive to "provide the conditions that make it conducive for workers to experience flow". To this end, we draw on concepts embedded in research on flow, technology and flow, and sociomateriality as described by Orlikowski [30] and Robey et al. [40] in order to theorize an alternate view of flow and technology, which we call technoflow. As opposed to other researchers in this area (e.g., Agarwal and Karahanna [1]), we do not assume that technology and flow are separate, but rather that technology and the individual experiencing flow are entangled, or "ontologically inseparable" [30]. We expound on this concept through a relational ontology, which "rejects the notion that the world is composed of individuals and objects with separately attributable properties that exist in and of themselves" [30].

This concept of technoflow can help researchers and managers better understand the social and material conditions related to why and how workers experience or do not experience flow, and to recognize technology's role in enabling or constraining it. We provide some brief directions for IS researchers and managers about how they can draw on this alternate view of technoflow through a hypothetical example of an office worker. We also revisit two contemporary theories in IS research-media synchronicity theory and technostress - and discuss how to incorporate technoflow using concepts central to those theories.

\section{Literature review}

\subsection{Flow}

Mihaly Csikszentmihalyi defines flow as "a mental state of extremely rewarding concentration that emerges in the space between frustration and boredom" [12, p. 347]. Csikszentmihalyi has developed his construct of flow over several decades. The most comprehensive theoretical construct conceptualized a flow experience as including the following nine dimensions: (1) challenge-skill balance; (2) action-awareness merging; (3) clear goals; (4) unambiguous feedback; (5) concentration on task; (6) sense of control; (7) loss of self-consciousness; (8) time transformation; and (9) autotelic experience [7].

The challenge-skill dimension is theoretically the most important to understanding the flow experience. Csikszentmihalyi explains this claim by discoursing that when an individual's skills are underutilized by an activity, boredom is the result. However, when one's skills are under-developed for the challenges of a specific activity, anxiety is the result. When one's skills are overly adequate for an activity such that the challenge is too low, boredom needs to be alleviated by engaging with more challenging activities or activity levels. On the other hand, when anxiety is created through one's skills being not sufficiently developed for the activity and the challenge is too great, one needs to downshift the level of challenge and enhance one's specific skills needed for a better alignment between skill and challenge level. In this way, meaningful and sustained growth, more frequent flow experiences, and ultimately self-transformation will occur. As such, matching one's skills to the challenges presented by a specific activity and modulating the skill-challenge dimension is of central importance to such growth. This is what Csikszentmihalyi [6] calls the "flow channel".

Csikszentmihalyi also notes that there are both individual and social factors that can interfere in one's ability to experience flow. An individual who is either overly self-conscious or self-centered will not have the control of consciousness necessary to experience flow very frequently or intensely. Likewise, both anomie and alienation can prevent one from experiencing flow because either one is too dependent on external factors such as unclear social expectations or overlydeterministic ones. The key at both the individual and collective level of experience is to avoid either fragmentation or excessive rigidity of attentional processes [7].

The ideal flow experience occurs when the individual seeks out autotelic experiences as well as to cultivate an autotelic self. An autotelic experience is one that is so consuming it becomes an end in itself; the extrinsic reward is not the goal. An autotelic self is developed by an attitude of engaging with any experience in a manner where one's focus is drawn into the undivided application of skill to challenge so as to attend to growth opportunities on a continual basis and thus every experience becomes intrinsically satisfying. 


\subsection{Measuring flow}

Empirically, a number of fields have adopted and extended Csikszentmihalyi's flow construct, including exercise and sport psychology $[18,19,20]$, education [47], and aging and quality of life [33]. Over time, multi-disciplinary scholarly teams have developed both qualitative and quantitative approaches to studying flow empirically. Csikszentmihalyi \& Larson [9] developed their experience sampling method (ESM) to further early theoretical work on flow. In one of the seminal qualitative studies of flow experience, Privette \& Bundrick [36] were able to differentiate flow experience from peak experience $(\mathrm{PE})$ and peak performance (PP). Subjects reported PE as fulfilling, significant, and spiritual whereas they reported PP as full focus and self in a clear process. Flow was differentiated from PP and PE by play and characterized by outer structure and the importance of other people.

Novak, Hoffman, and Young [29] developed a structural modeling approach to studying flow in online environments and were able to support and refine Hoffman \& Novak's [16] construct to include skill in Web use and challenges presented by that use as key antecedents to flow experience in online navigation. Based on the observation that many early empirical studies reduced their focus to the skillchallenge dimension of flow, Rheinberg, Vollmeyer, \& Engeser [38] developed a Flow Short Scale (FKS) with only 10 items to measure 6 of the construct's dimensions plus an additional 3 items to measure worry.

In addition to the FKS, there are two scales to measure flow that have emerged from the theoretical construct of flow. Both scales have been tested and refined so as to be valid and reliable measures. The first scale is the "flow state scale" (FSS-2). The second scale is the "disposition flow scale" (DFS-2). Each scale's original version was modified to improve the measurement of some dimensions of flow. In their second iteration, both scales have been subjected to confirmatory factor analyses of item analysis and cross-validation samples. Both of these scales held up and are valid and reliable self-measure instruments. Each one serves a different purpose in flow research. The FSS-2 is used to measure flow experienced within a particular highly physical event, primarily sportbased $[18,20]$. The DFS-2, on the other hand, is used to measure the frequency of flow experiences in a subject's chosen physical activity more generally [14].

Because the DFS-2 measures the relationship between a given individual and his/her reported experience of a flow state with a given physical or intellectual activity, the DFS-2 is a research instrument that has advanced the theoretical conceptualization of "flow" from a state-based physical experience to a trait-influenced phenomenon not limited to intense physical activities such as surfing, whitewater kayaking, or rock climbing.

\section{Technology and flow}

A number of scholars have applied Csikszentmihalyi's flow construct to the field of human-computer interaction [13, 46, 48]. For example, Hoffman and Novak (1996) defined a flow experience in an online environment as meeting the following criteria: (1) characterized by a seamless sequence of responses facilitated by machine interactivity; (2) intrinsically enjoyable; (3) accompanied by a loss of self-consciousness; and (4) self-reinforcing. The IS discipline has followed the lead of these researchers by considering flow as a measurable, objective concept that influences other empirical phenomena. Much of the research on flow in the IS discipline has focused on consumers and general technology use in contexts such as online shopping $[1,22,29]$, adoption of mobile streaming serves [21], acceptance and use of instant messaging services [23], and video game play [42].

In a recent literature review of flow in IS research, Rissler et al. [39] acknowledge four distinct streams of flow and technology. The first stream is championed by Jackson and March [20] and Ghani et al. [13]. Researchers in this stream position flow as comprised of several constructs consisting of several items derived from the nine dimensions of flow by Csikszentmihalyi. Authors in this stream have found that the challenge-skill balance, clear goals, and unambiguous feedback dimensions are antecedents of flow [39]. Moreover, authors in this stream have operationalized the autotelic experience (i.e., the desired outcome associated with flow) in terms of enjoyment or positive affect, and position it as a critical outcome variable.

A second stream involves work that is based on Agarwal and Karahanna [1]. In this stream of research, flow is conceptualized and operationalized in terms of cognitive absorption, which is based on five dimensions: curiosity, control, focused immersion, temporal dissociation, and heightened enjoyment [39]. In this way, flow is regarded as a second-order construct that includes such dimensions. Researchers in this stream have used cognitive absorption (as flow) in the context of the web, e-learning, and virtual worlds [39].

A third stream is based on the work of Webster et al. [48]. This stream regards flow as comprised of four 
measurable dimensions: control, attention, curiosity, and intrinsic interest. These conditions include the ability to feel in control during the interaction with technology, being able to focus one's attention to a narrow field of technological stimulus, being encouraged to satisfy curiosity through an exploration of technology, and experiencing intrinsic interest when using the technology [48].

The fourth stream of research on flow in IS research stems from Novak et al. [29]. Flow in this stream is treated as a uni-dimensional construct [39]. For example, when investigating users' interactions with using a website, Novak et al. [29] ask questions like "In general, how frequently would you say you have experienced "flow" when you use the Web?" In essence, the dimensions of flow are absent, and flow is regarded as a measurable construct in and of itself.

Overall, IS researchers typically regard flow and its relationship with technology in terms of a positivist approach using variance logic, whereby flow can serve as a causal determinant or outcome associated with technological use in multiple contexts $[27,31]$. In this sense, IS researchers have not focused on individual cases associated with technology and its role in achieving flow; rather, researchers have mainly generalized technology as a set of common features and empirical findings that can be reproduced statistically and are generalizable across contexts.

In the next section, we advocate for an alternative view of technology and flow.

\section{Revisiting flow: Technoflow}

The four streams of flow research discussed previously, while seemingly conceptually different, all share several fundamental assumptions about the nature of reality and the role and influence of technology in organizations. Ontologically, each stream of flow in IS research subscribes to the notion that technology and the flow experience are different, separate, and essentially context-free. IS researchers therefore assume that a generalizable technology experience (i.e., use, intention to use, continuance of use) can influence a generalizable flow experience, and vice versa. Orlikowski [23] refers to this as an "ontology of separateness." This is defined by Introna [17] as "a simple dualistic view of agency which claims that agency is located either in the human or in the artefact". We would like to represent this tradition of research on technology and flow (i.e., the four streams of research discussed above) through a hyphenated term: techno-flow. Under the ontology of separateness, we argue the hyphen symbolically separates the technology and flow.
Our call is to discuss a novel opportunity to dive deeper into how to conceptualize and understand the relationship between technology and flow through a non-hyphenated concept called technoflow. Technoflow, we argue, falls under a relational ontology [30], which assumes that individuals and the properties of the objects interacted are not distinct, but are rather entangled. Orlikowski [30] provides the following quote by Introna [17] to explain this concept more accurately:

"It would not be incorrect to say that our existence has now become so entangled with the things surrounding us (if it even makes sense to use the notion of 'surround') that it is no longer possible to say, in any definitive way, where we end and they begin, and vice versa. [ . . . ] We are the beings that we are through our entanglements with things - we are thoroughly hybrid beings, cyborgs through and through."

Under a relational ontology, researchers focus their inquiry on cases and attempt to identify how individuals and technologies are interrelated, or entangled, through the concept of practice. Practices are identified by researchers, who attempt to observe and recognize local, context-specific relationships that exist among individuals and objects (i.e., the social and the material). Researchers understand practices by identifying recurrent activities of the individual and material objects. The individuals and objects are not treated ontologically as separate, but as entangled, meaning that the individual and the material artifact, at the same time, possess relational attributes that shape how the individual (and the artifact) perform activities repeatedly.

Orlikowski and Scott [32] provide an example of identifying practices in an office setting. The authors describe an individual working in an office setting at one's desk. The individual sits at a desk in a chair and types with a keyboard while looking at information on a computer screen. The individual is also surrounded by several Post-It notes, pieces of paper, water bottles, etc. Each of these material objects, as well as the individual, possess attributes and logics imbued in them that shape a "pattern of workflow, ready to be actively configured into a situated work performance" [32].

In terms of technoflow, we argue that IS researchers, managers, and leaders of organizations should attempt to identify patterns imbued in the social and material in order to create the conditions that facilitate flow, or a series of peak experiences that engage employees in ways which both get the best out of employees while facilitating their own selfactualization. Following this logic, we argue that the ontological separateness associated with previous 
research which we classify as techno-flow has overlooked the potential to recognize and identify patterns associated with individual work practices so that workers can achieve flow experience. Below we provide an example of how researchers may accomplish this.

\section{Technoflow in practice: An example of an office worker}

Below, we briefly articulate how researchers and managers may recognize potential patterns associated with sociomaterial entanglement in a work context that is related to the kinds of flow experiences that facilitate engagement. To do so, we rely on four dimensions of flow that have been articulated in previous research on human-technology interaction: control, attention, curiosity, and intrinsic interest [48]. In this sense, we advocate for researchers to engage with existing literature and theories to identify principles related to the phenomenon under investigation (e.g., flow) so that its spirit can be both maintained and expounded upon. This is closely related to the concept of theoretical engagement [41], in which researchers draw on concepts embedded in existing theories to frame their research phenomena.

We articulate how researchers can use these four dimensions of flow (control, attention, curiosity, and intrinsic interest) as conceptual platforms for identifying sociomaterial properties and patterns in the context of office work. This example is based on a hypothetical office environment characterized by an individual sitting at a desk interacting with/using a computer, keyboard, and software to complete his/her work tasks.

Researchers should attempt to document the contextual practice associated with the type of task the individual is performing (e.g., emailing, making a presentation, typing a report), as well as the state of the material environment associated with the specific work practice (e.g., the type of software, the algorithms embedded in the software, the hardware, the materials on and surrounding the desk). This includes provision for any commonalities and differences of how the individual completes his or her work across employees. Researchers should also document the time and place of the work tasks, as well as the coworkers involved-given the work practice and entanglement of the social and material — and how the individual experiences flow, may be interdependent on other individuals at any given time and in a collocated or distributed space [32]. We provide more detailed examples below.

\subsection{Control}

Feelings of being in control of a situation is a central aspect of flow state achievement [48]. When identifying control in an office setting, under a specific work practice, researchers can seek out attributes and patterns that individuals develop and articulate in terms of control. For example, researchers can seek out patterns associated with the responsiveness and malleability of the artifact as related to specific work tasks, as well as the structure of the algorithm underlying the technology in use. Researchers may also want to distinguish several patterns and attributes that may relate to an individual feeling that the artifact is working for and with him/her, rather than vice versa.

Pilke [35] found, for example, that individuals articulated a flow experience in terms of the relationship with technology that provided immediate feedback. Conceptually, to identify specific aspects of the work practice that are related to control, researchers could draw on literature that defines control in terms of a few criteria: control of the interaction, a sense of control, and cognitive engagement [39].

Rather than only identifying conditions that may be related to achieving control, researchers and managers should document sociomaterial patterns and relationships that exhibit a lack of control, and can therefore postulate how to alleviate such more detrimental patterns. For example, previous research has found that continuous access to mobile device technology at work created feelings of loss of control over personal time and cognition [2, 3, 34]. Moreover, Mazmanian, Orlikowski, \& Yates [28] uncovered an "autonomy paradox" among knowledge workers using mobile devices, whereby continuous access led to "a collective reduction of autonomy as workers began to engage with work at all times."

As such, we suggest that a lack of control over the role of technology in one's work life, particularly as the number of and type of technological devices or software applications increases or becomes integral in completing one's work tasks, may be antithetical to experiencing increased engagement via flow state achievement. Such patterns that neglect control, such as the increased numbers of software, hardware, etc. may be noted in terms of achieving control.

\subsection{Attention}

The ability to focus one's attention on a task related to technology is another aspect of achieving flow [48]. For instance, in circumstances characterized by one individual and one device, individuals can become absorbed and even 
"mesmerized" through the computer technology interaction in a way indicative of flow [48]. The attention concept is related to focus immersion, which has been used in prior flow studies. Focused immersion requires that "all of the attentional resources of an individual are focused on the particular task, thereby reducing the level of cognitive burden associated with task performance" [1].

Researchers should attempt to document the social and material aspects related to attention in terms of the material artifacts predominant in keeping (and sustaining) an individual's attention. For example, researchers could document the material environment around the individual, as well as how the individual assembles his or her work environment and software in use. Researchers could also identify the key pieces of software that were present (e.g., a Word document) and likely more importantly not present during the individual focusing his or her attention, such as not having an email application open. Moreover, researchers should document the pieces of hardware surrounding the individual and note how the individual relates to such hardware. Pilke [35], for example, discovered that users achieved a flow state when using desktop computers, but not mobile devices.

In addition to identifying patterns associated with actualizing attention, researchers could note the patterns that interrupt an individual's attention. Today's knowledge worker is constantly inundated with multiple artifacts competing for attention. As such, this "hyper-connectivity" can likely be "distracting and overwhelming" and relate to "fragmented attention, reduced concentration and superficial thought processes" [21, p. 196].

As such, this may not actualize a flow state. Moreover, this inability to achieve the kind of focused attention needed for flow state achievement due to hyper-connectivity has been found to reduce employee engagement and worsen disengagement by exacerbating employee burnout, exhaustion, and job creep [24].

\subsection{Curiosity}

Flow state achievement is fostered via arousal of an "individual's sensory or cognitive curiosity" [48]. In contexts characterized by a single user interacting with a single technological device, it is easy to understand how this is true. Many will resonate with the scenario whereby one becomes absorbed in satisfying one's curiosity by exploring and experimenting with the various apps, menu options, functions, and possibilities of a technological device in a way indicative of flow. Researchers should document the contextual interplay governing the relationship with the artifact that may be articulated in terms of curiosity. This could be associated with simply the features of a software inexorably linked to the individual carrying out a task. For example, the software may be designed in a way that the individual can quickly maneuver among various dimensions within the software to find what he or she is searching for.

We also acknowledge that in the context of office work, one's relationship with the sensory and cognitive curiosity that technology may afford may hinder the ability to experience flow. An office worker, for example, may have a relationship in which the technology affords searching through social media. However, such activities may be involved in achieving a flow state, which is ultimately articulated by the individuals. Moreover, researchers should document the material artifacts surrounding the office worker, such as a smartphone and/or tablet. Such a secondary artifact may be related to a context that is outside of work, and therefore outside of the boundaries of the office context (e.g., one's personal and social life).

For example, researchers and managers may notice patterns related to notifications by the secondary device (e.g., text messages from friends, social media alerts) or those related to a personal hobby or interest (e.g., sports scores, news). Moreover, this toggling back and forth between technological stimuli and toggling back and forth between work, social, and personal interests may represent a form of "underengaged behavior" [21, p. 195], thereby delaying or hindering curiosity related to the task at hand. Again, researchers should document patterns associated with such relationships with primary and secondary devices and note if they are related to curiosity, and ultimately, flow.

\subsection{Intrinsic interest}

Flow state achievement also relies on the intrinsic interest of the activity to the user $[6,48]$. This means the user is engaging in "the activity for its own pleasure and enjoyment rather than for some utilitarian purpose" [41, p. 414]. Researchers should acknowledge the role of artifact in the activity itself, and focus on identifying patterns related to holding such interest in the individual. For example, an office worker may be tasked with crafting a presentation with a team of individuals. The office worker may have intrinsic interest in the subject and the presentation, and may be communicating with his or her team through the technology in order to complete the presentation, receive feedback, brainstorm ideas, etc.

In this sense, the user's relationship with the technology is not only one associated with using 
software to create the images and text in the presentation, but also communicating with team members, searching for information related to the content of the presentation, among other activities. Researchers and managers can then identify patterns associated with such interest, whether it be helping or hindering the intrinsic interest (and thus engagement) of the user.

\section{Recommendations for theorizing flow}

Inspired by Robey et al. [40], we provide two examples about how to revisit existing and contemporary IS theories to incorporate the relational ontological position of sociomateriality, and specifically, technoflow. In doing so, we argue that these theories have overlooked the material features of the technology. Below we explain how to revisit two theories and to incorporate technoflow: media synchronicity and technostress.

\subsection{Media synchronicity theory}

In 2008, Dennis, Fuller, and Valacich [10] provide a modernized view of the media richness theory, instantiated as the media synchronicity theory (MST). MST was theorized in response to the plethora of technology that exists in organizations, and conceptualizes a "fit" that needs to occur among individuals when sending and receiving information and comprehending situations and organizational issues. Specifically, MST "focuses on the ability of media to support synchronicity, a shared pattern of coordinated behavior among individuals as they work together" [10]. MST focuses on two primary processes governing communication among individuals: conveyance and convergence. Conveyance occurs when individuals use technology to process information in terms of creating and revising a mental model of the situation. Convergence occurs when individuals interpret the situation, not the information, which often requires a back and forth transmission of information in order to reach a mutual understanding of the situation [10].

The conveyance and convergence processes have different technological requirements for information transmission and processing among individuals, and for synchronicity among individuals to occur (i.e., a shared understanding, or "fit", among the processes and technology used). In other words, for individuals to come to a shared understanding of information, tasks, roles, and responsibilities within an organization, they need to select the proper technology in order to fit the information that needs to be conveyed or converged upon in a given situation. If a fit occurs, a shared understanding occurs, and the media has been synchronized to fit the needs of the communication patterns associated with the task [10].

Unfortunately, MST treats the materiality of technology as conceptually vague, in that the media (i.e., technology) is treated as an exogenous force that impacts how individuals send and receive information. In doing so, MST exists in an ontology of separateness that treats the technology and the information and the individuals as separate and provides for a limited understanding of the social and material conditions that are related to synchronicity.

We argue that several important concepts embedded in MST prove fruitful for investigating technoflow and understanding the sociomaterial conditions that may or may not be related to flow. Under a modified version of MST, IS researchers can use the principle of theoretical engagement [41] to draw on the two central processes of conveyance and convergence, as well as the concept of synchronicity, to document the attributes and patterns of individuals related to how synchronicity occurred, and the materiality present for it to occur. For example, researchers can draw on convergence to understand how individuals communicated back and forth to arrive at a shared meaning by documenting how the social and material objects were enacted through evolving sociomaterial conditions throughout the convergence process. In essence, convergence could be used to conceptually map the sociomaterial entanglement to illustrate how convergence occurred over time (or did not).

Researchers can extend MST into technoflow by incorporating dimensions of technoflow (e.g., control, attention, curiosity, intrinsic interest) in conjunction with conveyance and convergence to recognize patterns of sociomaterial interactions among individuals and groups. For example, drawing on convergence, researchers can emphasize the functions that the material objects provided in terms of actualizing convergence and the sociomaterial patterns associated with convergence. More detail about the contextually social and material interplay around synchronicity and its relationship with flow can then be further understood.

\subsection{Technostress}

Technostress is commonly defined as "a modern disease of adaptation caused by an inability to cope with new computer technologies in a healthy manner" [4]. In IS research, technostress has been operationalized as an aggregate of five technostress creators: techno-overload, techno-invasion, technocomplexity, techno-insecurity, and techno-uncertainty 
[37, 43]. Like much research in the IS discipline, the technology in technostress is treated as an exogenous force that induces several individual and organizational outcomes. For example, technostress has been shown to increase role stress [44], lower innovation [45], and reduce job satisfaction, organizational commitment, and continuance commitment [37]. In this vein, the social and material conditions remain absent from much research on technostress, as does a conceptual and empirical link between technostress and flow.

Technoflow and technostress are two research streams that have the potential for seamless integration. However, the two have not been conceptually linked in IS research, and technostress has not been instantiated through a relational ontology. We therefore advocate for researchers to revisit technostress under a relational ontology. To accomplish this, researchers can draw on concepts embedded in contemporary technostress literature (e.g., Tarafdar et al. [43]), such as the technostress creators, and use these concepts as a theoretical frame to understand and document the material objects related to technostress. For example, one technostress creator commonly studied in technostress research is called techno-overload [44]. Techno-overload involves technology inducing feelings of excess work in terms of the information and in learning new features and new software packages. Specifically, techno-overload "describes situations where the use of computers forces people to work more and work faster" [44]. However, unfortunately, the situations in the literature have not been well articulated or documented conceptually or empirically. This, we argue, is due to the nature of the ontological positioning underlying the majority of technostress research in the IS discipline (e.g., ontological separateness). Repositioned under a relational ontology, researchers can document the social and material conditions that may be related to the contextual situations that individuals undergo when experiencing stress related to technology. An insight into how such situations related to stress materialize should be valuable information for managers to have.

In addition, following the lead of Hargrove, Nelson, and Cooper [15], as well as recent literature by Tarafdar et al. [43], we recognize that technostress can be thought of in terms of eustress (good stress) and distress (bad stress). Hargrove et al. [15] argue that there are "positive possibilities of good stress," and advocate for a connection among eustress and flow. The authors cite research by Daniel Goleman who has shown that when individuals experience flow their brain scans show positive emotions, such as those related to eustress (e.g., positive affect, hope, meaningfulness). However, the authors do not provide much guidance on how to connect technostress and flow.

We therefore advocate for IS researchers fill this gap by considering technostress as a holistic process comprised of techno-eustress and techno-distress, and to craft research designs that connect technostress and flow. Using the conceptualization of technoflow under a relational ontology, researchers could detail the social conditions and material objects that are related to individuals expressing feelings associated with eustress, and document the sociomaterial patterns that actualized such eustress. Moreover, researchers can recognize the patterns that exist when such feelings of eustress are then articulated under the dimensions associated with flow. Since there may be some conceptual overlap in eustress and flow, we thereby advocate for researchers using existing theoretical frames to conceptualize the experiences and emotions related to technostress and flow.

\section{Conclusion}

Multi-faceted arrays of technology are increasingly integrated into organizations and the work lives of employees. At the same time, both organizational performance and employee well-being are hinged upon the engagement of workers. By using a relational ontology that assumes the constitutive entanglement of individuals and technology, we argue that facilitating employee flow states with technology which cultivate the autotelic self can be achieved by understanding the concept of technoflow. As opposed to treating individuals, technology, and other work life artefacts as separate and distinct, technoflow integrates provision for context-specific practices imbued with the social and material which create the conditions necessary for flow state achievement. Using four dimensions flow (i.e., control, attention, curiosity, and intrinsic interest) that have been articulated in previous research [48], we identified opportunities for exploring and understanding such practices in a typical organizational setting. We also suggest how two existing IS theories (i.e., MST and technostress) can utilize the relational ontology of technoflow to better integrate provision for the sociomaterial conditions that may or may not be related to flow. We feel this represents a fruitful conceptual in-road for new explorations into the human - technology nexus of organizational life.

\section{References}


[1] Agarwal, R., and E. Karahanna, "Time Flies When You're Having Fun: Cognitive Absorption and Beliefs about Information Technology Usage", MIS Quarterly 24(4), 2000, pp. 665-694.

[2] Barker, J.R., "Tightening the Iron Cage: Concertive Control in Self-Managing Teams", In C. Grey and H. Willmott, eds., Critical Management Studies: A Reader. Oxford University Press, 2005.

[3] Barley, S.R., and G. Kunda, Gurus, Hired Guns, and Warm Bodies: Itinerant Experts in a Knowledge Economy, Princeton University Press, 2006.

[4] Brod, C., Techno Stress: The Human Cost of the Computer Revolution, Addison-Wesley, 1984.

[5] Choi, D.H., J. Kim, and S.H. Kim, "ERP training with a web-based electronic learning system: The flow theory perspective", International Journal of Human-Computer Studies 65(3), 2007, pp. 223-243.

[6] CSIKSZENTMIHALYI, M., "Beyond Boredom and Anxiety", Experiencing Flow in Work and Play, 1975.

[7] Csikszentmihalyi, M., Flow: The Psychology of Optimal Experience, New York, Harper \& Row, 1990.

[8] Csikszentmihalyi, M., Good Business: Leadership, Flow, and the Making of Meaning, Penguin, 2004.

[9] Csikszentmihalyi, M., and R. Larson, "Validity and Reliability of the Experience-Sampling Method", In M. Csikszentmihalyi, ed., Flow and the Foundations of Positive Psychology: The Collected Works of Mihaly Csikszentmihalyi. Springer Netherlands, Dordrecht, 2014, $35-54$.

[10] Dennis, A.R., R.M. Fuller, and J.S. Valacich, "Media, Tasks, and Communication Processes: A Theory of Media Synchronicity”, MIS Quarterly, 32(3), 2008, pp. 575-600.

[11] Finneran, C.M., and P. Zhang, "A person-artefacttask (PAT) model of flow antecedents in computermediated environments", International Journal of HumanComputer Studies 59(4), 2003, pp. 475-496.

[12] Gallup, Employee engagement on the rise in the U.S, 2017.

[13] Ghani, J.A., R. Supnick, and P. Rooney, "The Experience of Flow in Computer-mediated and in Face-toface Groups", Proceedings of the Twelfth International Conference on Information Systems, University of Minnesota (1991), 229-237.

[14] Giffin, L.B., B.R. Payne, and E. Stine-Morrow, "Dispositional Flow: A Resource for Successful Aging", Gerontologist, (2013), 476-476.
[15] Hargrove, M.B., D.L. Nelson, and C.L. Cooper, "Generating eustress by challenging employees: Helping people savor their work", Organizational Dynamics 42(1), 2013, pp. 61-69.

[16] Hoffman, D.L., and T.P. Novak, "Marketing in Hypermedia Computer-Mediated Environments: Conceptual Foundations", Journal of Marketing 60(3), 1996, pp. 50-68.

[17] Introna, L.D., "Maintaining the reversibility of foldings: Making the ethics (politics) of information technology visible", Ethics and Information Technology 9(1), 2007, pp. 11-25.

[18] Jackson, S.A., and R.C. Eklund, "Assessing Flow in Physical Activity: The Flow State Scale-2 and Dispositional Flow Scale-2", Journal of Sport and Exercise Psychology 24(2), 2002, pp. 133-150.

[19] Jackson, S.A., S.K. Ford, J.C. Kimiecik, and H.W. Marsh, "Psychological Correlates of Flow in Sport", Journal of Sport and Exercise Psychology 20(4), 1998, pp. 358-378.

[20] Jackson, S.A., and H.W. Marsh, "Development and Validation of a Scale to Measure Optimal Experience: The Flow State Scale", Journal of Sport and Exercise Psychology 18(1), 1996, pp. 17-35.

[21] Jung, Y., B. Perez-Mira, and S. Wiley-Patton, "Consumer adoption of mobile TV: Examining psychological flow and media content", Computers in Human Behavior 25(1), 2009, pp. 123-129.

[22] Koufaris, M., “Applying the Technology Acceptance Model and Flow Theory to Online Consumer Behavior", Information Systems Research 13(2), 2002, pp. 205-223.

[23] Lu, Y., T. Zhou, and B. Wang, "Exploring Chinese users' acceptance of instant messaging using the theory of planned behavior, the technology acceptance model, and the flow theory", Computers in Human Behavior 25(1), 2009, pp. 29-39.

[24] MacCormick, J.S., K. Dery, and D.G. Kolb, "Engaged or just connected? Smartphones and employee engagement", Organizational Dynamics 3(41), 2012, pp. 194-201.

[25] Mahnke, R., A. Benlian, and T. Hess, "Flow Experience in Information Systems Research: Revisiting its Conceptualization, Conditions, and Effects”, ICIS 2014 Proceedings, 2014.

[26] Mahnke, R., A. Benlian, and T. Hess, A Grounded Theory of Online Shopping Flow, Darmstadt Technical University, Department of Business Administration, 
Economics and Law, Institute for Business Studies (BWL), 2015.

[27] Markus, M.L., and D. Robey, "Information Technology and Organizational Change: Causal Structure in Theory and Research", Management Science 34(5), 1988, pp. 583-598.

[28] Mazmanian, M., W.J. Orlikowski, and J. Yates, "The Autonomy Paradox: The Implications of Mobile Email Devices for Knowledge Professionals", Organization Science 24(5), 2013, pp. 1337-1357.

[29] Novak, T.P., D.L. Hoffman, and Y.-F. Yung, "Measuring the Customer Experience in Online Environments: A Structural Modeling Approach", Marketing Science 19(1), 2000, pp. 22-42.

[30] Orlikowski, W.J., "The sociomateriality of organisational life: considering technology in management research", Cambridge Journal of Economics 34(1), 2010, pp. 125-141.

[31] Orlikowski, W.J., and C.S. Iacono, "Research Commentary: Desperately Seeking the 'IT' in IT Research-A Call to Theorizing the IT Artifact", Information Systems Research 12(2), 2001, pp. 121-134.

[32] Orlikowski, W.J., and S.V. Scott, "The entanglement of technology and work in organizations", 2008.

http://www.lse.ac.uk/collections/informationSystems/

[33] Payne, B.R., J.J. Jackson, S.R. Noh, and E.A.L. StineMorrow, "In the zone: Flow state and cognition in older adults", Psychology and Aging 26(3), 2011, pp. 738-743.

[34] Perlow, L.A., "Boundary Control: The Social Ordering of Work and Family Time in a High-Tech Corporation", Administrative Science Quarterly 43(2), 1998, pp. 328357.

[35] Pilke, E.M., "Flow experiences in information technology use", International Journal of Human-Computer Studies 61(3), 2004, pp. 347-357.

[36] Privette, G., "Peak experience, peak performance, and flow: A comparative analysis of positive human experiences", Journal of Personality and Social Psychology 45(6), 1983, pp. 1361-1368.

[37] Ragu-Nathan, T.S., M. Tarafdar, B.S. Ragu-Nathan, and Q. Tu, "The Consequences of Technostress for End Users in Organizations: Conceptual Development and Empirical Validation", Information Systems Research 19(4), 2008, pp. 417-433.

[38] Rheinberg, F., R. Vollmeyer, and S. Engeser, "Die Erfassung des Flow-Erlebens",
[39] Rissler, R., M. Nadj, and M. Adam, "Flow in Information Systems Research: Review, Integrative Theoretical Framework, and Future Directions", Wirtschaftsinformatik 2017 Proceedings, 2017.

[40] Robey, D., B. Raymond, and C. Anderson, "Theorizing Information Technology as a Material Artifact in Information Systems Research", In P.M. Leonardi, B.A. Nardi and J. Kallinikos, eds., Materiality and Organizing. Oxford University Press, 2012, 216-236.

[41] Sarker, S., X. Xiao, and T. Beaulieu, "Guest Editorial: Qualitative Studies in Information Systems: A Critical Review of Some Guiding Principles", Management Information Systems Quarterly 37(4), 2013, pp. iii-xviii.

[42] Seger, J., and R. Potts, "Personality Correlates of Psychological Flow States in Videogame Play", Current Psychology 31(2), 2012, pp. 103-121.

[43] Tarafdar, M., C.L. Cooper, and J.-F. Stich, "The technostress trifecta - techno eustress, techno distress and design: Theoretical directions and an agenda for research", Information Systems Journal, 29(1), 2019, pp. 6-42.

[44] Tarafdar, M., Q. Tu, B. Ragu-Nathan, and T. RaguNathan, "The Impact of Technostress on Role Stress and Productivity", J. Manage. Inf. Syst. 24(1), 2007, pp. 301328.

[45] Tarafdar, M., Q. Tu, and T. Ragu-Nathan, "Impact of Technostress on End-User Satisfaction and Performance", J. Manage. Inf. Syst. 27(3), 2010, pp. 303-334.

[46] TREVINO, L.K., and J. WEBSTER, "Flow in Computer-Mediated Communication: Electronic Mail and Voice Mail Evaluation and Impacts", Communication Research 19(5), 1992, pp. 539-573.

[47] Vollmeyer, R., and F. Rheinberg, "Motivational Effects on Self-Regulated Learning with Different Tasks", Educational Psychology Review 18(3), 2006, pp. 239-253.

[48] Webster, J., L.K. Trevino, and L. Ryan, "The dimensionality and correlates of flow in human-computer interactions", Computers in Human Behavior 9(4), 1993, pp. 411-426. 